\title{
LA RESOLUCIÓN DE PROBLEMAS: UNA ESTRATEGIA PARA APRENDER A APRENDER
}

\begin{abstract}
Resumen
En este artículo el autor presenta a consideración de la comunidad académica una serie de elementos que permiten mostrar la importancia de enseñar centrados en resolución de problemas que fomente en los alumnos la capacidad de aprender a aprender. En el se integran: antecedentes, puntos de vista teóricos, análisis de concepciones y tipos de problemas que pueden plantearse en el aula, buscando interesarlos en lo que constituye la resolución de problemas, en la perspectiva del desarrollo de una pedagogía en la que el alumno sea el gestor de su propio aprendizaje.
\end{abstract}

Palabras clave: resolución de problemas, aprender a aprender.

\section{PROBLEMS SOLVING. A STRATEGY FOR LEARNING TO LEARN}

\begin{abstract}
In this article, the author presents in consideration of the academic community, a series of elements that allows showing the importance to teach focused on problems resolutions that promote the capacity to learn how to learn in students. In it, antecedents, points of view, analysis of conceptions and kind of problems are tackled, being thought about in the classroom, looking for interesting them in what constitutes the resolution of problems; in the perspective of the development of a pedagogy where the students are the agents of their own learning.
\end{abstract}

Key words: Problems resolution, learn to learn.

\section{INTRODUCCIÓN}

De los muchos propósitos que animan los cambios educativos en los ámbitos nacional y mundial, tal vez el que mejor resume el espíritu psicopedagógico es el de fomentar en los alumnos la capacidad de aprender a aprender. En las diversas etapas y áreas se destaca la necesidad de que los alumnos adquieran el conjunto de conocimientos ya elaborados que constituye la cultura y la ciencia de nuestra sociedad y, de modo muy especial, que adquieran habilidades y estrategias que les permitan aprender a aprender por sí mismos nuevos conocimientos. En la perspectiva de una sociedad muy flexible en las demandas laborales y culturales a sus ciudadanos, al tiempo que muy competitiva, no basta con proporcionar saberes empaque-

* Doctorando en Educación. Profesor de la Universidad Pedagógica Nacional, Bogotá. oviedopaulo@gmail.com. Texto original recibido: 04-2-07 y aprobado: 20-4-07. 
tados, cerrados en sí mismos, sino que hay que hacer de los alumnos personas capaces de enfrentarse a situaciones y contextos cambiantes, que exijan de ellos aprender conocimientos y habilidades nuevas. Por ello, los alumnos que hoy aprendan a aprender estarán previsiblemente en mejores condiciones de adaptarse a los cambios culturales, tecnológicos y laborales que los esperan.

En este sentido, uno de los vehiculos más asequibles para llevar a los alumnos a aprender a aprender es la resolución de problemas. Frente a una enseñanza basada en la transmisión de conocimientos, la resolución de problemas puede constituir un contenido educativo y todo un enfoque o un modo de concebir las actividades educativas. La resolución de problemas se basa en el planteamiento de situaciones abiertas y sugerentes que exijan de los alumnos una actitud activa y un esfuerzo por buscar sus propias respuestas, su propio conocimiento. La enseñanza basada en la resolución de problemas supone fomentar en los alumnos el dominio de procedimientos, así como la utilización de los conocimientos disponibles para dar respuesta a situaciones cambiantes y distintas. Así, enseñar a los alumnos a resolver problemas supone dotarlos de la capacidad de aprender a aprender, en el sentido de habituarlos a encontrar por si mismos respuestas a las preguntas que los inquietan o que necesitan responder, en lugar de esperar una respuesta ya elaborada por otros y transmitida por el libro de texto o por el profesor.

\section{ANTECEDENTES}

La búsqueda de calidad en la educación a llevado a numerosos investigadores a desarrollar diversos estudios, los cuales pueden enmarcarse en dos grandes corrientes o tendencias.

La primera tendencia se refiere a las investigaciones orientadas a estudiar cómo aprenden los es- tudiantes a resolver problemas, ésta es una actividad permanente del campo de la psicología. Según Anderson ${ }^{1}$, la finalidad de estas investigaciones consiste en conocer como patrones empíricos los modos procedimentales de los expertos al resolver problemas, y establecer sus diferencias con los novatos. Una vez conocidos, estos procedimientos se emplean en procesos de enseñanzaaprendizaje.

El conocimiento de los procedimientos utilizados por expertos y novatos, así como el funcionamiento de las memorias a largo y a corto plazos y de la memoria sensorial, tienen un valor intrinseco evidente. No obstante, de acuerdo con los planteamientos de algunos investigadores, entre los cuales se destaca Selvaratnam ${ }^{2}$, los modelos de resolución de problemas elaborados a partir de esas investigaciones presentan limitaciones claras: no son apropiados para resolver problemas abiertos; las estrategias didácticas implícitas que propugnan se enmarcan en el paradigma de enseñanza-aprendizaje por transmisión verbal y tienen por objeto reproducir los procedimientos exitosos, lo cual no favorece el desarrollo de la creatividad ni el ejercicio del pensamiento divergente.

La segunda tendencia incluye una serie de investigaciones orientadas a determinar cómo enseñan los profesores, a fin de analizar los procedimientos empleados cuando se enseña a resolver problemas $y$, al mismo tiempo, a plantear alternativas más acordes con los procesos de construcción científica; es una actividad de la didáctica de las ciencias que ha ocupado a un importante número de investigadores en los años recientes ${ }^{3}$.

\footnotetext{
$1 \mathrm{~J}$. Anderson, Cognitive Psychology and Its Implications, New York: W. H. Freeman and Co., 1990.

${ }^{2} \mathrm{M}$. Seivaratnam, Problem-solving a model approach, Education in Chemistry. nov. de 1990, pp. 163-165.
}

Como características básicas de estas últimas investigaciones se tienen, por un lado, tomar como posibilidades de resolución los rasgos esenciales de los contenidos establecidos y los procedimientos de las ciencias naturales a la hora de resolver problemas de investigación; y, por otro, orientar la enseñanza hacia modelos de resolución de problemas coherentes con la creatividad del trabajo científico, tratando de solucionar situaciones problemáticas, abiertas e interesantes para los alumnos y favoreciendo el pensamiento productivo, tanto en el proceso de resolución de los problemas como en todas las actividades de aprendizaje de las ciencias.

La preocupación general estriba en posibilitar que profesores y estudiantes experimenten una evolución determinada como consecuencia de los procesos educativos; en este sentido, el problema que se plantea es definir qué características de la enseñanza permiten que esta posibilidad se convierta en realidad. En consecuencia, numerosos equipos de profesores han hecho propuestas metodológicas para enseñar a resolver problemas con esta orientación $n^{4}$.

La riqueza de la investigación en el campo de la resolución de problemas ha generado, según Marín ${ }^{5}$, tres enfoques: a) enseñanza para la resolución de problemas; b) enseñanza sobre la resolución de problemas; y c) enseñanza mediante la resolución de problemas.

${ }^{3}$ C. Furio, et ál. Contribución de la resolución de problemas como investigación al paradigma constructivista de aprendizaje de las ciencias, Investigación en la Escuela, vol. 24, pp. 89-99, 1994.

4 J. Martinez-Torregrosa, La resolución de problemas de fisica como investigación: un instrumento de cambio metodológico, Tesis doctoral, Universidad de Valencia, 1987

${ }^{5}$ N. MARín, Condiciones fundamentadas de enseñanza-aprendizaje para la resolución de problemas en ciencias, 1996. 
Las pretensiones en cada uno de los enfoques mencionados son diferentes y pueden justificar una doble función de los problemas en la enseñanzaaprendizaje: en unos casos, los problemas como justificación de los conocimientos adquiridos, en contraposición con la visión de los problemas como dinamizadores de adquisición de conocimientos; en otros casos, se reconocen paradigmas diferentes en la resolución de problemas, entendidos como formas distintas de entender la resolución de problemas según su función en la enseñanza-aprendizaje. En este sentido, este escrito se ha organizado en torno a cinco capítulos: el primero, referido a los antecedentes; el segundo, a los puntos de vista teóricos que han fundamentado la resolución de problemas; el tercero, al análisis de los conceptos de problema y su resolución; el cuarto, a los tipos de problemas; y el quinto, a la resolución de problemas como una actividad de investigación.

\section{PUNTOS DE VISTA TEÓRICOS QUE HAN FUNDAMENTADO

Las investigaciones sobre resolución de problemas se han realizado fundamentalmente al amparo de la psicología experimental; sin embargo, en las últimas décadas ha tomado cuerpo la investigación específica de resolución de problemas en el marco de las ciencias naturales (física, quimica, biologia), así como en el de las matemáticas y el de las ciencias sociales.

El abordaje se hace respetando el orden cronológico de surgimiento de las distintas teorías que han acogido la resolución de problemas, así: la conductista, la de la Gestalt, la asociativa, la del procesamiento de la información, la piagetiana, y la del constructivismo.

- En la teoría conductista, en el proceso de resolución de pro- blemas lo más importante es la respuesta y su mecanismo de selección, asociados con el estímulo presente en el problema. En esta corriente las primeras investigaciones se basaron en la determinación de las estrategias de resolución de problemas empleadas por distintas personas a través de la observación, en un intento de buscar similitudes entre ellas. Así, Watts ${ }^{6}$ identificó cuatro etapas en el proceso: a) la preparación (acumulación de información); b) la incubación (marginación transitoria del problema); c) la iluminación (un darse cuenta repentino); d) la verificación (hallazgo de la solución).

En esta misma línea pueden situarse los trabajos de Polya ${ }^{7}$ sobre resolución de problemas matemáticos. La principal novedad es la de apuntar que una estrategia adecuada para resolver problemas considerados muy difíciles consiste en su fraccionamiento en subproblemas más simples, que si admiten una solución.

- Coincidiendo con las observaciones de Polya, aunque precursores en el tiempo, los gestaltistas detectaron la tendencia de los solucionadores a fraccionar los problemas en diversas etapas para intentar resolverlas posteriormente. La mayor contribución de esta teoría ha sido el énfasis puesto en la vertiente perceptual del proceso; para dichos seguidores, la aprehensión apropiada de las partes del problema asegura que las fuerzas de la organización produzcan de algún modo la solución; sin embargo, no especifican con exactitud qué son esas fuerzas de la organización.

${ }^{6} \mathrm{M}$. WatTS, The science of problemsolving. A practical guide for science teachers, London: Cassell, 1991.

${ }^{7} \mathrm{G}$. PolyA, On solving mathematical problems in high school, In S. Krulik and R. E. Reys (eds.), Problem solving in school mathematics, Reston, Virginia, 1980.
Desde esta corriente de pensamiento el fracaso en la resolución de problemas se debe frecuentemente a la persistencia de un escenario (set) rígido e inadecuado, que puede estar causado por la experiencia previa o por la forma en que se expresan las instrucciones del problema. Ese escenario (set) podría desvanecerse mediante orientaciones del experimentador que permitan concentrar o dirigir la atención hacia los aspectos más significativos del problema.

Otra contribución novedosa de los teóricos de la Gestalt es la "valoración" de las posibles soluciones de un problema. Esto tiene una importancia especial en la vida cotidiana, así como en la toma de decisiones, donde diversas soluciones generan ventajas e inconvenientes. Desde esta perspectiva, la resolución de problemas se concibe de modo invariable como un proceso productivo.

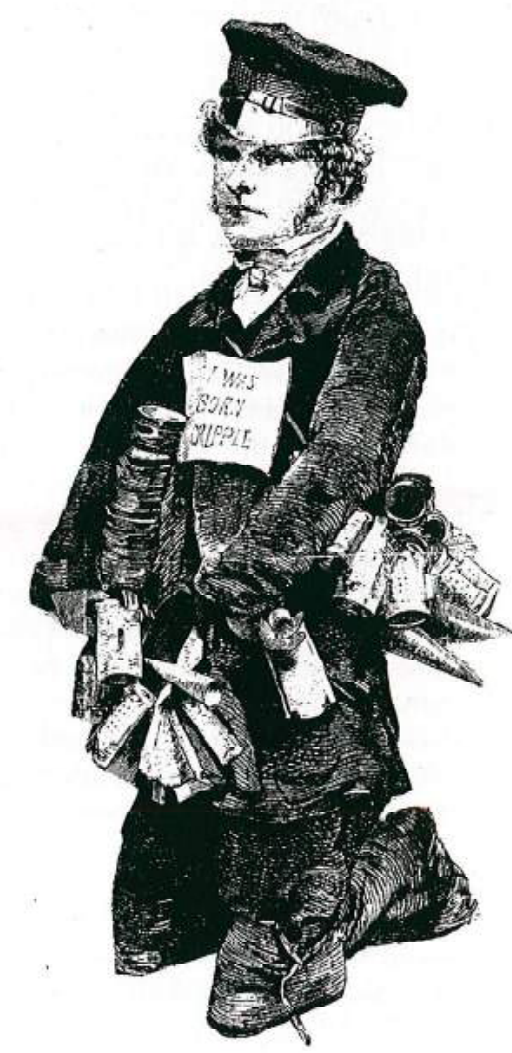


- En la teoría asociativa la característica esencial es la jerarquía de respuesta. Ello se refiere a la noción de que un estímulo está asociado con varias respuestas y de que la fuerza de la asociación varía. Estas respuestas pueden estar ordenadas en cuanto a su fuerza, formando una jerarquía. Existe un problema cuando la respuesta más fuerte es incorrecta y la solución implica la activación de respuestas sucesivas de la jerarquía hasta que se provoca la respuesta correcta.

La esencia de la teoria asociativa consiste en que puede describirse, en forma adecuada, la solución de problemas como asociaciones estímulo-respuesta, incluidas las conexiones mediadoras (respuesta mediadora-estímulo mediador); su establecimiento y su operación están gobernados por los principios del condicionamiento. Kendler ha señalado que la solución de problemas incluye un proceso horizontal (encadenamiento de asociaciones en el transcurso del tiempo) y un proceso vertical (operación simultánea de asociaciones múltiples). El análisis de la predisposición se relaciona con el proceso horizontal, ya que está dirigido hacia el orden temporal de las respuestas en una situación problema ${ }^{8}$.

- La teoría del procesamiento de la información describe la resolución de problemas como una interacción entre el sistema de procesamiento de la información del sujeto y un ambiente de la tarea como la describe el experimentador. Este enfrentamiento produce en el solucionador una representación mental del problema, denominada "espacio del problema", que contiene el estado actual del problema, el estado final o meta y todos los estados

\footnotetext{
${ }^{8}$ A. Newell y H. A. Simon, Human Problem-Solving. Prentice-Hall Inc., Engle-
} wood Cliffs, N. J., 1972. intermedios. La resolución de un problema conduce a un proceso de búsqueda dirigida por el objetivo a través del espacio del problema $^{9}$.

- Según la teoría de Piaget, el individuo que pudiera acceder a las operaciones formales sería capaz de resolver cualquier tipo de problema, independientemente de su contenido. No obstante, años más tarde se reconoce la influencia del contenido en la resolución de problemas formales, y se deriva en la perspectiva piagetiana o pospiagetiana que pone su interés en la necesidad de potenciar el desarrollo cognitivo a través de la resolución de problemas ${ }^{10}$.

- El punto de partida de la toma de posición del constructivismo, en el seno de la resolución de problemas, es posible analizarlo en la dependencia entre dicho proceso y el contenido en el que se contextualiza el problema. Se confirma así que el razonamiento tiene forma y contenido ${ }^{11}$. Lo novedoso de esta teoría estriba en el estudio de modelos de pensamiento circunscritos a situaciones específicas de los problemas. Así, se ha llegado a considerar la resolución de problemas independientemente de su estructura lógica pero con gran énfasis en su representación mental, en su comprensión por parte del sujeto $y$, en definitiva. en las ideas previas de éste sobre los conceptos implicados.

En cierta forma, esta tendencia

9 Ibid.

${ }^{10} \mathrm{~J}$. POMEs, La metodología de resolución de problemas y el desarrollo cognitivo: un punto de vista postpiagetiano, Enseñanza de las Ciencias, 9(1), pp. 78-82, 1991.

${ }^{11}$ L. J. Pozo, "Las ideas del alumnado sobre la ciencia: de dónde vienen, a dónde van... y mientras tanto qué hacemos con ellas", Alambique, 7, pp. 18-28, 1996. ha convergido con la otra que se deriva de la psicología del procesamiento de la información, en cuanto a que ésta ha abordado el diseño de sistemas expertos que tratan de solucionar problemas específicos. Así mismo, se ha acometido la comparación entre la resolución de problemas por parte de expertos y novatos, es decir, entre sujetos que difieren en la calidad y la cantidad de sus preconcepciones $^{12}$.

En resumen, los investigadores han tendido a adoptar una de estas teorías hacia la resolución de problemas. Sin embargo, al considerarlas se infiere que hay tres puntos de vista sobre dicho proceso. Una orientación perceptual, que conduce a explicaciones teóricas que ponen énfasis en la reorganización de la situación problema por quien soluciona el mismo, la utilización de explicaciones introspectivas y el interés en el efecto de las variaciones, durante la presentación del problema, antes que en la dificultad de la solución. En contraste evidente, las descripciones asociativas de los problemas que se hacen en términos impersonales de estímulos y respuestas, el interés se centra en el estudio de las variaciones en la fuerza asociativa, utilizando conceptos de aprendizaje como reforzamiento, extinción y generalización. Las características de la resolución de problemas como proceso de búsqueda resultan del intento de descubrir las estrategias que puede emplear quien lo resuelve, al seleccionar y evaluar las soluciones alternativas.

Los puntos de vista teóricos no son contradictorios, por lo cual es innecesario elegir uno y excluir los demás; sin embargo, cada investigador debe tener claridad acerca de cómo asume la resolución de problemas; los diversos énfasis en los puntos de vista señalados quizá reflejen las diferentes concepciones acerca de lo

${ }^{12}$ A. Newell y H. A. Simon, op. cit. 
que es importante en este proceso. También existe la posibilidad, de que un punto de vista particular presente más ventajas para estudiar cierto tipo de problemas.

\section{ANÁLISIS DE ALGUNAS CONCEPCIONES DE PROBLEMA Y RESOLUCIÓN}

Al revisar la literatura existente, se han encontrado concepciones y clasificaciones muy diversas, teóricamente justificables a partir de los criterios escogidos para tales fines.

En un intento de combinar la tradición gestaltiana (por ejemplo, de los trabajos de Duncker ${ }^{13}$ ) con la tradición asociacionista (de los trabajos de Newell y Simon $\left.{ }^{14}\right)$, que se tratarán más adelante, Garret ${ }^{15}$ define el problema como una "situación donde el paradigma existente no puede aplicarse y para la cual incluso puede no existir solución; las situaciones donde se conoce o asume que pueden resolverse con un paradigma dado recibirian la denominación puzzle". Con esta concepción el problema constituye un proceso productivo, mientras que el puzzle corresponde a una situación cuya solución se alcanzará con un procedimiento meramente reproductivo.

Asi mismo, Garrett sostiene que "cada individuo, dependiendo de su conocimiento personal y de las estrategias o recursos de que disponga, verá una situación dada como un problema o como un puzzle". De este modo, el que una situación pueda considerarse o no como problema es algo totalmente subjetivo.

\footnotetext{
${ }^{13} \mathrm{~K}$. Duncker, "On problem solving". Psychological Monographs, 58(270), 5, 1945.

${ }^{14}$ A. Newell y H. A. Simon, op. cit.

${ }^{15}$ R. M. GARREt, Resolución de problemas y creatividad: Implicaciones para el currículo de ciencias, Enseñanza de las Ciencias, 6(3), 224-230, 1988.
}

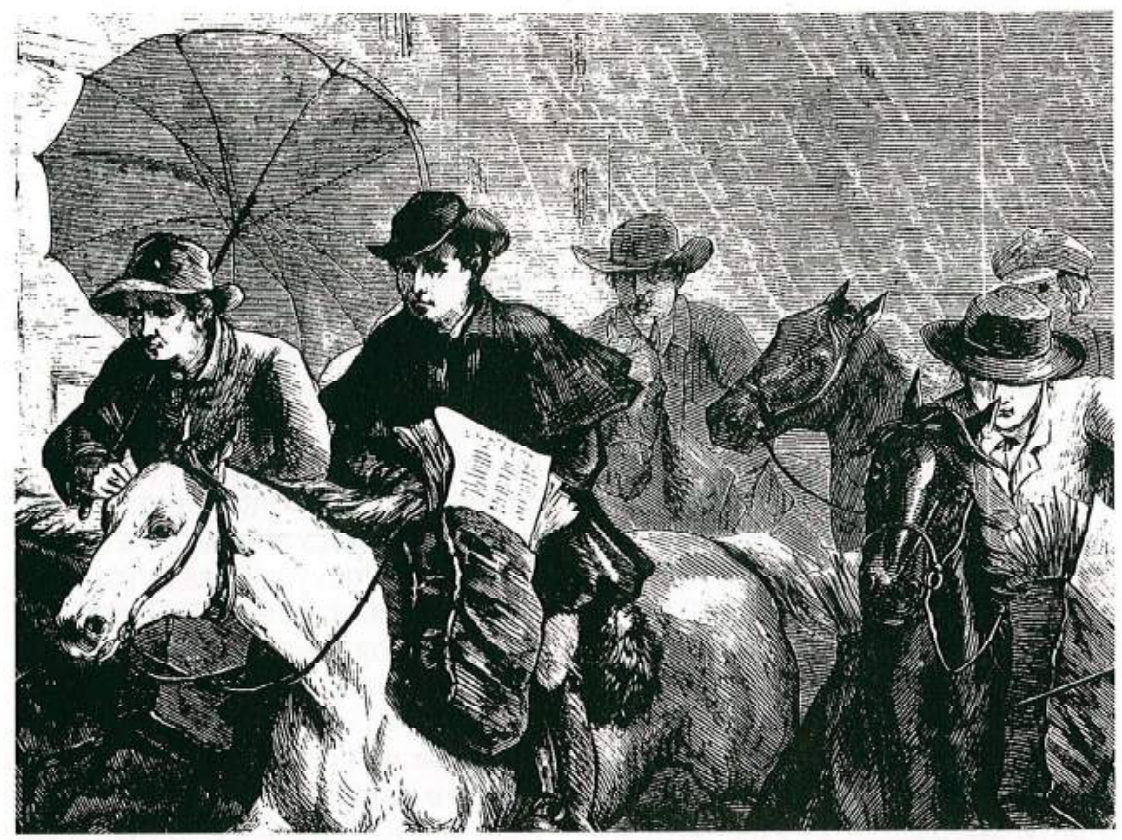

Otros autores (Woods, Crowe, Hoffman y Wright ${ }^{16}$ ) definen el problema como una situación estimulante para la cual el individuo no tiene respuesta; es decir, el problema surge cuando el individuo no puede responder de manera inmediata y eficaz a una situación. Según Hudgins ${ }^{17}$, un problema es una situación que presenta dificultades para las cuales no hay soluciones evidentes. Para MartínezTorregrosa ${ }^{18}$ las situaciones planteadas en el aula como problemas (por resolver con lápiz y papel) no se consideran problemas, puesto que se conoce su solución. Sin duda es cierto, desde un punto de vista psicológico, que cuando una persona encuentra la forma de resolver un problema, éste deja de existir.

\footnotetext{
${ }^{16}$ D. R. Woods, C. M. Crowe, T. W. HoffMAN Y J. D. WRIGHT, Challengs to Teaching Problem-Solving Skills. Chem. 13 News Waterloo University, 155(1), 1985.

${ }^{17}$ B. B. Hudgins, Cómo enseñar a resolver Problemas en el Aula, Buenos Aires: Paidós, 1996.

${ }^{18} \mathrm{~J}$. MARTINEZ-TORREGrosa, La resolución de problemas de física como investigación: un instrumento de cambio metodológico, Tesis doctoral, Universidad de Valencia, 1987.
}

Sin embargo, en el contexto del aula el profesor sigue hablando de problemas, tanto si sabe como si no sabe resolverlos. Más aún: en numerosos casos sólo cuando ha resuelto un problema sabe si es o no adecuado para ser utilizado como herramienta de acceso al conocimiento. El profesor basa la elección de una situación (problema) determinada, como forma de facilitar la comprensión, en las características del proceso de resolución de esta situación. Por ello se piensa que basar una definición en un hecho totalmente subjetivo puede resultar demasiado ambiguo y originar problemas de difícil solución y que en el contexto educativo el problema, como elemento de una estrategia de enseñanza, debe definirse en sí mismo por las etapas que comporta su proceso de resolución y por la complejidad que presente para la persona que afronta tal situación.

El problema, en el contexto del aula y como componente base de una estrategia de enseñanza fundada en su resolución, puede definirse como una situación cuya solución requiere que el sujeto analice unos hechos y desarrolle en forma razonada una estrategia que le permita obtener unos datos (numéricos o 
no), procesarlos (relacionarlos entre sí y con los hechos), interpretarlos y llegar a una conclusión (respuesta). Este análisis y este razonamiento deben basarse en la comprensión del tema o del campo al que pertenece la situación. Un problema no podrá ser resuelto mediante el recuerdo, el reconocimiento, la reproducción o la aplicación de un único algoritmo. De este modo, el problema vendrá definido por el proceso de resolución que deberá seguir la persona que intenta alcanzar su solución y por el grado de dificultad que presente para esa persona.

Teniendo en cuenta lo anterior, y con el propósito de participar en el debate sobre qué es resolver un problema, Jessup et ál. ${ }^{19}$, entendemos por problema "una situación, espontánea o prevista, para la cual no se tiene una solución eficaz y adecuada de manera inmediata, hecho que si bien produce incertidumbre, se convierte en una potencialidad para resolverla, debido a que moviliza a quien la enfrenta hacia la búsqueda de solución. Su carácter de problema está dado de modo fundamental por la posibilidad de resolverla o solucionarla según los desarrollos quien lo resuelve, asi como por el proceso que implica su resolución"

En cuanto a la resolución, según Frazer $^{20}$, es un proceso que utiliza el conocimiento de una disciplina, sus técnicas y habilidades de esa disciplina, para salvar el espacio existente entre el problema y su solución. Efectivamente: la resolución de problemas podría concebirse como un proceso que conduce a una serie de actividades, cuyo fin es la consecución de la solución. Tal definición resultaría aplicable a cualquier dis-

${ }^{19} \mathrm{C}$. Jessup et ál, Resolución de problemas y enseñanza de las ciencias, Líneas de investigación, Doc. mim., pp. 6-8, 1997.

${ }^{20}$ R. M. Frazer, Solving Chemical Problems. Chemical Society Review, 11(2), 171-190, 1982. ciplina de las ciencias naturales. Sin embargo, el modelo de definición no establecería las condiciones internas que se desarrollan en el sujeto que resuelve el problema

Kempa ${ }^{21}$ considera la resolución de problemas como un proceso de información sucede en el cerebro del sujeto y que implica y requiere varias funciones de su memoria (memoria a corto plazo, memoria de trabajo y memoria a largo plazo). Según este modelo, el estudiante lee el problema, lo interpreta en cuanto a tareas que se solicitan y a ideas fundamentales que se requieren y selecciona los métodos, las estrategias y los hechos que pueden conducirlo a la solución. Por tanto, el sujeto ha de haber comprendido el problema. Éste es, quizás, uno de los puntos más controvertidos del proceso de la resolución de problemas, que además se ha incluido en el significado de la palabra "problema".

Según Novak y Gawing 22 , la resolución hace referencia al proceso mediante el cual una situación incierta es clarificada, por la aplicación, en mayor o menor medida, de conocimientos y procedimientos por parte del sujeto, asi como a la reorganización de la información almacenada en la estructura cognitiva, es decir, a un aprendizaje.

En sintesis, aunque existen algunos estudios sobre el proceso de resolución de problemas, la mayor parte de las investigaciones ha limitado sus observaciones a la comparación de la dificultad de los problemas en diferentes condiciones. Se establece la dificultad del problema por la probabilidad de solución o por el tiempo de solución; sin embargo, se

${ }^{21}$ R. F. KEMPA, "Resolución de problemas de química y estructura cognoscitiva", Enseñanza de las Ciencias, 4(2), 99110, 1986

22 J. Novak y D. Gowing, Aprendiendo a Aprender, Barcelona: Martínez-Roca, 1988. requiere que, cuando entre distintas condiciones difiera la dificultad del problema, tal diferencia pueda observarse en las probabilidades de solución rápida. Se ha interpretado de manera característica que la dificultad de solución refleja el obstáculo de producción de la respuesta, al suponer que las instrucciones han preparado al sujeto de manera adecuada, y asi se presta poca atención a la evaluación de las soluciones potenciales. Finalmente, parecería que la elección de las variables y las tareas experimentales por parte de un investigador depende de su concepto de resolución de problemas, según si su descripción enfatiza la percepción, el alertamiento asociativo o el procesamiento de información.

En esos términos Jessup et ál. ${ }^{23}$ entenderíamos por resolución "un proceso mediante el cual una persona que se enfrenta a un problema trata de identificarlo, de delimitarlo, de explorar posibilidades de resolverlo, de elegir las estrategias adecuadas para lograrlo a partir de sus desarrollos individuales, de llevarlas a la práctica mediante la aplicación de métodos y técnicas apropiadas y de obtener cierta aproximación a la solución del mismo".

\section{ALGUNAS POSICIONES ACERCA DE TIPOS DE PROBLEMAS}

Aunque los problemas no están clasificados en categorías definitivas, las características que establecen algunos autores sólo constituyen un medio de tener una idea de la clase de problemas diseñados por los diferentes teóricos e investigadores para explicar e investigar su solución.

Frazer $^{24}$ establece una diferencia entre problemas artificiales y problemas reales. El problema artificial es aquel cuya solución es conocida

${ }^{23}$ Op. cit.
${ }^{24}$ Op. cit. 
por la persona que lo ha presentado (el profesor o el autor del libro de texto). Este tipo de problemas puede clasificarse, en función de la naturaleza de la solución, como: problema "cerrado", con una solución única, y problema "abierto", con un número variable de soluciones. El problema real, es aquel para el cual no se conoce la solución, incluso puede que ésta no exista. En consecuencia, la categoría "problemas verdaderos", de Garrett, corresponde a la de "problemas reales" presentada por Frazer. Sin embargo, este último autor establece para los problemas reales dos subcategorias, las de problemas reales con objetivo o sin objetivo dirigido en función de que estén o no encaminados a resolver algún aspecto concreto de interés científico, tecnológico o social.

Según este autor, en la enseñanza de las ciencias, en general, los problemas utilizados son de tipo artificial y, salvo raras excepciones, cerrados. Este tipo de problemas se usa con dos propósitos: facilitar la comprensión al estudiante, haciéndole utilizar su propio conocimiento, y prepararlo para la resolución de problemas reales.

Garrett ${ }^{25}$ distingue entre ejercicios puzzles o rompecabezas (potencialmente resueltos dentro de un paradigma, y que a su vez, pueden ser abiertos, por existir para ellos varias respuestas adecuadas, o ser cerrados, cuando sólo existe una respuesta correcta) y problemas verdaderos, para los cuales puede no existir respuesta, sino algunos grados de comprensión. En este sentido, los primeros implican reconocimiento y reproducción, mientras que los segundos suponen un proceso de construcción.

Watts ${ }^{26}$ distingue dos tipos de problemas: a) los PS1 (problem-solving 1), que incluyen los más tradicionales, asociados a los problemas académi-

\footnotetext{
${ }^{25}$ Op. cit.

${ }^{26}$ Op. cit.
}

cos de las clases de ciencias, cuyos enunciados están bien definidos y cuya resolución se basa en procesos puramente intelectuales; y b) los PS2 (problem-solving 2), más genéricos, generalmente cualitativos y próximos a la vida real, que pueden requerir práctica experimental y cuyo objetivo fundamental es el desarrollo de estrategias de resolución, implicando los ámbitos cognitivo, de manipulación y afectivo.

Perales $^{27}$ establece tres categorías, organizadas con base en diferentes criterios: a) según el campo de conocimiento aplicado, distingue los cotidianos y los académicos; b) según el tipo de tarea, agrupa los cuantitativos, que demandan determinaciones numéricas, y los cualitativos, más centrados en la interpretación científica de los fenómenos reales; y c) en cuanto a la naturaleza del enunciado y a las características del proceso de resolución, diferencia los problemas cerrados, fácilmente resolubles mediante la utilización de algoritmos determinados, de los abiertos, que exigen la utilización del pensamiento productivo para el diseño de estrategias de resolución.

Caballer y Oñorbe ${ }^{28}$ distinguen tres tipos de problemas: a) "problemascuestiones", para reforzar y aplicar la teoria; b) "problemas-ejercicios", para el aprendizaje de técnicas de resolución ya establecidas que permitan alcanzar una solución correcta; y c) "problemas-investigación", entre las cuales se incluirian también actividades de laboratorio, útiles para el desarrollo de procedimientos y actitudes hacia la ciencia y sus métodos de trabajo.

${ }^{27} \mathrm{~F}$. J. Perales, "La resolución de problemas: Una revisión estructurada", Enseñanza de las Ciencias, 11(2), 170$178,1993$.

${ }^{28} \mathrm{M}$. J. Caballer y A. ONoRbe, Resolución de problemas y actividades de laboratorio en la enseñanza y el aprendizaje de las ciencias de la naturaleza, Barcelona: ICE Universidad de Barcelona- Horsori, 1997.
En consecuencia, no existe acuerdo sobre una clasificación. Sin embargo, las caracterizaciones hechas por Caballer y Oñorbe, Perales, Watts, Garrett y Frazer $^{29}$ constituyen un medio de tener una idea de las clases de problemas que existen; para investigar su solución Jessup et ál. ${ }^{30}$ consideran que "valdría la pena plantearse un nuevo criterio como tipo de aproximación para su resolución. En este sentido, tendríamos una nueva clasificación en términos de: problemas teóricos, cuando su solución no implica aproximaciones de corte experimental, y problemas experimentales, cuando su solución requiere de la experimentación".

Las clasificaciones presentadas, que son tan sólo algunas de las posibles, ponen claramente de presente que en esta materia queda aún mucho trabajo por realizar y constituye un buen tema de trabajo puntual.

\section{LA RESOLUCIÓN DE PROBLEMAS COMO INVESTIGACIÓN}

Según los estudios de Newell y Simon $^{31}$, cuando el alumno inicia el proceso de resolución de un problema definido, crea un "espacio del problema" interno, es decir, una comprensión personal del problema. Esta fase del proceso implica la reformulación del problema en unos términos en los cuales el sujeto comprende, estableciendo interrelaciones entre el problema y su conocimiento personal, incluyendo las similitudes existentes con problemas previamente resueltos y extractando los componentes más importantes del problema como vía para facilitar la selección y la ejecución de los siguientes pasos. El reconocimiento de problemas similares es valioso en particular, puesto que proporciona la

\footnotetext{
${ }^{29}$ Ver la bibliografia al final del artículo.

${ }^{30} \mathrm{Op}$. cit.

${ }^{31}$ Op. cit
} 
puesta en marcha de experiencias y procedimientos conocidos (hechos, algoritmos y heurísticas), que serán la guia de la etapa siguiente de resolución. Algunos aspectos de esta etapa se han denominado "redescripción", puesto que la persona, a menudo, abstrae hechos notables del problema y los describe en una forma diferente. La mayor parte de los ejemplos, en este sentido, está relacionada con la resolución de problemas en fisica, aunque existen observaciones en el área de la genética que apoyan este hecho. El desarrollo de un espacio del problema inicial propicio depende claramente del uso adecuado de una base de conocimiento propio.

La planificación de la estrategia de resolución lleva asociadas algunas preguntas: ¿Qué información poseo acerca del problema? ¿Qué nueva información necesito? ¿Qué secuencia de acciones debo seguir? ¿Cómo puedo encontrar lo que necesito saber acerca de ...? ¿Cómo saber cuando he resuelto el problema? La información que el alumno posee acerca del problema estará almacenada en su memoria semántica. La utilidad de la información dependerá de cómo ésta haya sido organizada y de cómo pueda ser recuperada para afrontar las diversas tareas.

En tal sentido, la práctica común de la enseñanza de las ciencias naturales y de la biología en España, por ejemplo, ha prestado gran atención a la organización del conocimiento. Los contenidos de los programas por lo general son presentados a los alumnos de modo secuencial, capítulo por capítulo, de forma que ellos deben tratar de integrar todos los conocimientos acumulados y organizados coherentemente para facilitar su uso.

Una vez elaborada la secuencia de pasos por seguir para lograr la solución, la obtención de datos constituirá una parte integral de cualquier modelo de resolución. En esta etapa, el alumno se pregunta: ¿Qué nueva información necesito?; podrá necesitar datos cualitativos o cuantitativos. El proceso de obtención de datos dependerá en gran medida del proceso de reflexión, en tanto que la validez de los datos obtenidos dependerá del grado de exactitud y precisión ejercido por la persona que los obtiene.

Una vez conseguida la información necesaria, ésta se organiza. EI alumno establece algunos patrones para ordenar e integrar los datos obtenidos, buscando respuesta a la pregunta ¿Cómo puedo organizar la información de forma útil? La forma elegida por éste será considerada provisional o temporal. Si durante el desarrollo posterior del proceso de resolución éste descubre que su elección no es adecuada, necesitará modificar o incluso desechar la estrategia de resolución inicial.

El siguiente paso, tanto en el modelo desarrollado por el Departamento de Educación del Estado de Nueva York (análisis de los datos) como en el de Murphy y Gott (interpretación de los datos), requiere que el alumno establezca las relaciones entre los datos, reconozca las características que proporcionan las relaciones causaefecto y haga constar las deducciones posibles. El análisis de los datos debe ser cuidadoso y razonado para asegurar una resolución eficaz. Este análisis lleva al alumno a obtener conclusiones o a elegir posibles opciones que lo lleven a la solución.

Por último, la toma de decisiones y la evaluación completan el proceso de resolución. Esta etapa final lleva asociadas preguntas como: ¿Qué decisiones debo tomar? ¿Cuáles han sido las alternativas elegidas y por qué? ¿Cuáles son las consecuencias de cada alternativa? ¿El proceso seguido ha sido adecuado? ¿La solución obtenida tiene sentido? Si no se ha logrado la solución, la evaluación del proceso permitirá deducir el camino a seguir decidiendo la validez de cada uno de los pasos seguidos durante la resolución.
Según Gil y Martínez-Torregrosa ${ }^{32}$ el problema es definido a través dé un proceso que se inicia partiendo de una experiencia y concluye con la formulación de unas preguntas. La respuesta a estas preguntas se logrará al finalizar el proceso de resolución. Este tipo de trabajo en educación resultaría de gran valor para aproximar al estudiante a la metodología de trabajo científico, pues tendría que emitir hipótesis, diseñar experimentos, realizarlos y analizar con rigor los resultados, que a su vez coadyuvan en la superación de errores conceptuales. De acuerdo con los planteamientos, esto posibilita un cambio conceptual profundo.

Murphy y Gott ${ }^{33}$ han analizado el proceso de resolución seguido por alumnos de trece y quince años de edad, respectivamente y han identificado una serie de etapas comunes en el proceso, esto los ha llevado a la construcción del modelo cíclico de resolución de problemas, que comprende las etapas de: Problema (definición e identificación) y formulación (en forma abierta, decidiendo qué se va a medir); diseño de un experimento (estableciendo condiciones); desarrollo del experimento (utilizando aparatos, realizando medidas y observaciones); recolección de datos (en tablas, gráficos, etc.); interpretación de datos obtenidos y extracción de conclusiones; evaluación (de resultados, métodos); y solución (respuesta).

El modelo cíclico es un proceso reiterativo que implica que el alumno realiza una evaluación, a distintos niveles, durante la búsqueda de la solución. Esto le permite rechazar

${ }^{32}$ D. GIL y JOAQUin MARTINEZ-TORREgROSA, Tres paradigmas básicos en la enseñanza de las ciencias, Enseñanza de las Ciencias, 1, 26-33, 1983.

${ }^{33}$ P. Murphy y R. Gott, citados en A. F. Sigüerza y M. J. Saez, Análisis de la resolución de problemas como estrategia de enseñanza de la Biología, Enseñanza de las Ciencias, 8(3), 223-230, 1990.1990. 
algunas de sus conclusiones y tomar nuevas decisiones. El modelo aproxima el proceso de investigación científica a la resolución de problemas en el aula, contemplando el aprendizaje como un proceso discontinuo de construcción y comprobación de hipótesis. En función de las hipótesis comprobadas, la persona que resuelve el problema intenta crear una secuencia de actuaciones que seguirá hasta que ésta resulte apropiada para lograr la solución. La secuencia creada mediará entre el estímulo (el problema) y la respuesta (la solución). Esta visión de aprendizaje se opone a la concepción asociacionista de la teoría de la continuidad y puede resultar apropiada para describir el proceso de pensamiento que tiene lugar en el periodo piagetiano de "operaciones formales".

Sigüenza y Sáez ${ }^{34}$, manifiestan que en la práctica educativa, con frecuencia pueden presentarse fundamentalmente dos tipos de aproximación del estudiante a los problemas: a) en muchos casos, los problemas son definidos exclusivamente por el profesor, b) su enunciado figura en un libro de texto. Sin embargo, existe un tercer tipo de acercamiento, aunque menos usual: el alumno, dirigido por el profesor, puede participar en la definición del problema. A juicio del equipo del cual forma parte el autor, una estrategia de enseñanza basada en la resolución de problemas deberia contemplar la redefinición del problema en el aula. De hecho, desde hace algunos años existen programas de educación general básica que contemplan la definición del problema y su resolución como componentes básicos de la estrategia de enseñanza de las ciencias naturales. Por ejemplo, el programa Elementary Science Syllabus, desarrollado por el Departamento de Educación del Estado de Nueva York para impartir enseñanza en el área de ciencias naturales desde la edad de cuatro años, propone un modelo

${ }^{34}$ A. F. Sigüenza y M. J. SaEz, op. cit. de definición y resolución de problemas esquematizado, en tres etapas: la primera, de definición del problema (por medio de un proceso que se inicia partiendo de una experiencia y concluye con la formulación de unas preguntas); la segunda, de proceso de resolución (que incluye acciones de planeación, obtención de datos, organización de datos, análisis de datos, generalización y/o síntesis a partir de los datos, y toma de decisiones); la tercera, es la solución (la respuesta a las preguntas).

Este modelo de resolución, aunque puede ser aplicado por los alumnos desde los cuatro años de edad, resulta especialmente apropiado en el periodo piagetiano de "operaciones concretas" (de siete a once años de edad). Superada esta etapa, la estructura cognitiva del alumno permite el uso de los modelos de destrezas y la experiencia de cada uno influirá en la estrategia de resolución. Así surgirán en ellos distintas formas de alcanzar la solución.

\section{CONCLUSIÓN}

La enseñanza basada en la solución de problemas supone fomentar en los alumnos el dominio de las habilidades y estrategias que les permitan aprender a aprender, así como la utilización de los conocimientos disponibles para dar respuesta a situaciones cambiantes y distintas.

El estudio en torno a la enseñanza y el aprendizaje de la resolución de problemas debe, en Matemáticas, propugnar por superar el enfoque tradicional a partir del análisis de sus diversos significados. La resolución de problemas abre paso a la distinción entre ejercicios y problemas en el contexto del aula, uno de los problemas recurrentes en la solución de los mismos. En Ciencias Naturales, debe abogar por la renovación y el enriquecimiento del concepto de problema para promover cambios conceptuales, metodológicos y actitudinales en los alumnos, ya que la resolución de problemas en el aula de ciencias tradicionalmente ha estado ligada bien a la realización de ejercicios cuantitativos o a trabajos prácticos de laboratorio; y en Ciencias Sociales, debe enfatizar la necesidad de abandonar la enseñanza exclusivamente transmisiva y la conveniencia de presentar sus contenidos de un modo más abierto a la exploración, el debate y la indagación, puesto que la naturaleza específica de los problemas sociales y de sus procesos de solución hace necesario no sólo reflexionar sobre las características específicas de este tipo de problemas, sino también sobre los tipos de actividades de aula que pueden utilizarse de modo más eficaz para enfrentar a los alumnos a la solución de problemas sociales.

Debemos tener presentes tres ideas: a) enseñar a resolver problemas en cada una de las áreas supone concentrarse en la enseñanza de los procedimientos, sin perder de vista los conceptos y las actitudes; b) diferenciar en el aula lo que es un verdadero problema de un ejercicio; c) cuál es el papel del profesor en la construcción de estrategias de solución de problemas por parte de los alumnos.

El alumno es el gestor de su propio aprendizaje, puesto que se enfrenta a una situación dada a partir de sus propios intereses y expectativas, teniendo como elemento fundamental, para resolverla, sus características individuales, desarrollando así mismo habilidades que le serán de utilidad en cualquier actividad de su vida, puesto que el enfrentarse a problemas es una actividad cotidiana para el ser humano.

El acercamiento a los procesos de indagación científica mediante la resolución de problemas, con el consiguiente desarrollo de habilidades básicas, procedimentales e investigativas, se constituye en una opción eficaz para incrementar la creatividad y la capacidad crítica de los alumnos, lo cual se constituye, a su vez, en fundamento de la autonomía y la responsabilidad social. 


\section{BIBLIOGRAFÍA}

Anderson, J., Cognitive Psychology and Its Implications, New York: W. H. Freeman and Co., 1990

Caballer, M. J. y Oñoree, A., Resolución de problemas y actividades de laboratorio en la enseñanza y el aprendizaje de las ciencias de la naturaleza, Barcelona: ICE Universidad de Barcelona- Horsori, 1997.

DUNCKER, K., "On problem solving". Psychological Monographs, 58(270), 5. 1945.

Fernandez GonzAlez, J. y EloRtegui EsCARTIN, N., ¿Qué piensan los profesores acerca de cómo se debe enseñar? Enseñanza de las Ciencias, 14(9), 331342, 1996.

Furıo, C., et ál. Contribución de la resolución de problemas como investigación al paradigma constructivista de aprendizaje de las ciencias, Investigación en la Escuela, 24, 89-99, 1994.

FrAzer, R. M., Solving Chemical Problems. Chemical Society Review, 11(2), 171-190, 1982.

GARRET, R. M., Resolución de problemas y creatividad: Implicaciones para el currículo de ciencias, Enseñanza de las Clencias. 6(3), 224-230, 1988.

GIL, D. y Martinez-Torregrosa, Joaguin, Tres paradigmas básicos en la enseñanza de las ciencias, Enseñanza de las Ciencias 1, 26-33, 1983.

Gil, D., Dumas, A., Caillot, M., MartinezTorREgrosa, J., y Ramirez, L., La resolución de problemas de lápiz y papel como actividad de investigación, Investigación en la Escuela, 6, 3-19, 1988.

Hudgins, B. B., Cómo enseñar a resolver Problemas en el Aula, Buenos Aires, Paidós, 1996.
Jessup, C., Margie N. Resolución de problemas y enseñanza de las ciencias, Líneas de investigación, Doc. mim., pp. 6-8, 1997.

Jessup, C., et ál. "La resolución de Problemas y la Educación en Ciencias Naturales", Ponencia Primer Encuentro de Facultades de Educación, Santafé de Bogotá, 2000.

KEMPA, R. F., "Resolución de problemas de quimica y estructura cognoscitiva", Enseñanza de las Ciencias, 4(2), 99110, 1986.

MARIN, N. Condiciones fundamentadas de enseñanza-aprendizaje para la resolución de problemas en ciencias, 1996.

Martinez P., J. A. Un problema planteado como actividad de investigación: estudio de las posibles trayectorias para el lanzamiento efectivo de un tiro libre de baloncesto. Enseñanza de las Ciencias, 18(1), 131-140, 2000.

MARTINEZ-TORREGROSA, J. La resolución de problemas de física como investigación: un instrumento de cambio metodológico. Tesis doctoral, Universidad de Valencia, 1987.

MetTes, et ál. Teaching and Learning Problem-Solving in Science Part I: A general strategy, Journal of. Chemical Education, 57, 882-885, 1980.

Newell, A. y Simon, H. A., Human Problem-Solving. Prentice-Hall Inc., Englewood Cliffs, N. J., 1972.

NovaK, J. y Gowing, D. Aprendiendo a Aprender, Barcelona: Martínez-Roca, 1988.

Perales Palacios, F. J. "La resolución de problemas: Una revisión estructurada", Enseñanza de las Ciencias, 11(2), 170$178,1993$.
POLYA, G. On solving mathematical problems in high school, In S. Krulik and R. E. Reys (eds.), Problem solving in school mathematics, Reston, Virginia, 1980 .

POMEs, J., La metodologia de resolución de problemas y el desarrollo cognitivo: un punto de vista postpiagetiano, Enseñanza de las Ciencias, 9(1), 78-82, 1991.

Pozo, J. I., "Las ideas del alumnado sobre la ciencia: de dónde vienen, a dónde van... y mientras tanto qué hacemos con ellas", Alambique, 7, 18-28, 1996.

Ramirf7 Castro, Juan Lorfenzo. La resolución de problemas de física y química como investigación en la enseñanza media: Un instrumento de cambio metodológico, tesis doctoral, Universidad Autónoma de Barcelona, Facultad de Ciencias Químicas, 1990.

Selvaratnam, M., Problem-solving a model approach, Education in Chemistry. nov. de 1990, pp. 163-165.

Siguenza, A. F. y Saez, M. J. Análisis de la resolución de problemas como estrategia de enseñanza de la Biología, Enseñanza de las Ciencias, 8(3), 223-230, 1990.

WATTS, M. The science of problem-solving. A practical guide for science teachers, London: Cassell, 1991.

Woods, D. R. ; Crowe, C. M.; Hoffman, T. W. y WRIGHT, J. D., Challengs to Teaching Problem-Solving Skills. Chem. 13 News Waterloo University, 155(1), 1985. 